\title{
Extracting Depth Information Using a Correlation Matching Algorithm
}

\author{
Mahmoud Abdelhamid, Jeffery Beers, Mohammed Omar* \\ Clemson University International Center for Automotive Research CU-ICAR, Greenville, USA. \\ Email: *momar@clemson.edu
}

Received February $14^{\text {th }}, 2012$; revised March $16^{\text {th }}, 2012$; accepted April $20^{\text {th }}, 2012$

\begin{abstract}
This manuscript presents a modified algorithm to extract depth information from stereo-vision acquisitions using a correlation based approaches. The main implementation of the proposed method is in the area of autonomous Pick \& Place, using a robotic manipulator. Current vision-guided robotics is still based on a priori training and teaching steps, and still suffers from long response time. This study uses a stereo triangulation setup where two Charged Coupled Devices CCDs are arranged to acquire the scene from two different perspectives. The study discusses the details to calculate the depth using a correlation matching routine which programmed using a Square Sum Difference SSD algorithm to search for the corresponding points from the left and the right images. The SSD is further modified using an adjustable Region Of Interest ROI along with a center of gravity based calculations. Furthermore, the two perspective images are rectified to reduce the required processing time. The reported error in depth using the modified SSD method is found to be around $1.2 \mathrm{~mm}$.
\end{abstract}

Keywords: Stereo-Vision; Depth; Passive Triangulation; Pick and Place

\section{Introduction}

Stereo vision systems are constructed to mimic the way human see objects in the 3D space while getting a sense of their depth. The human brain receives the two perspective images from the two eyes and processes or combines them to make one single mental image of a scene called cyclopean image [1]. The cyclopean view is a view constructed by the coherence-network; that is acquired by either eye. However, it is still controversial how the brain computes depth [2].

$3 \mathrm{D}$ imaging is playing bigger role in creating the interface between machines (mobile robotics, gaming consoles) and their surroundings and users. The process of combining images from two or more sources, either biological or manmade, resulting in a 3D image is called stereovision, which can usefully define [3] as directed at understanding and analyzing the 3D perception of objects based on imaging data. The sequence of steps in stereovision have commonly the following phases [3]; firstly is the image acquisition done by a dual camera system or a dynamic camera providing two perspective images. Secondly is the camera modeling step, and thirdly, is correspondence analysis. The last processing step is the triangulation that is the geometric technique of depth measurement.

\footnotetext{
"Corresponding author.
}

The structure of presented manuscript starts with citing prior work, while discussing the previous studies and their relevance to proposed method. Section three exposes the experimental work details for finding the depth using stereo vision setup. Section Four discusses the results and the proposed pick and place application using this approach. Finally, the study findings are summarized in the conclusion.

\section{Literature Review}

Different techniques and mathematics have been proposed and employed to approach the accurate depth extraction from a stereovision systems. The prior works will be categorized according to the four phases describe previously.

The image acquisition step depends on the sensing element that is the focal plane array spatial (pixel size) and temporal (acquisition rate) resolutions. While the camera modeling, also called camera calibration, defines the internal camera geometric and optical characteristics (intrinsic parameters) and the 3D position and orientation of the camera frame relative to a certain world coordinate system (extrinsic parameters) [4]. This step represents the relation between 3D world coordinate and image coordinates 2D. The main calibration techniques reported in open literature can be divided into three categories: First, reference object based on 3D calibration where the 
camera calibration is performed by observing a calibration object whose geometry in 3D space is known with precision, allowing for efficient calibration [5], Tsai [4] used this method via a calibration object that consisted of orthogonal planes; this implementation relied on simple theory and computation to provide accurate results; however it is still experimentally expensive due to its elaborate setup. Second calibration approach is done by Zhang in [6] through observing a planar pattern at different orientations. This calibration method will be used through proposed study because of its relative experimental ease and accuracy. Third calibration technique is based on a Plane calibration 1D method, which is relatively new technique proposed by Zhang in [7] Plane calibration 1D object relied on a set of collinear points, e.g., two points with known distances, or three collinear points with known distances. The Camera can be calibrated by observing a moving line around a fixed point, e.g. a string of balls hanging from the ceiling; this allows the calibration of multiple cameras at once. This method is good for a network of cameras mounted apart from each other, where the calibration objects are required to be visible simultaneously.

The research done on the correspondence analysis is based on biological principles; mainly the principle of retinal disparity. Two main classes of algorithms are reported. The first is based on matching; in this technique the comparison is done between the intensity values of a pixel in one image to intensity of pixels in another image. A point of interest is chosen in one image called window then a cross-correlation measure is used to search for a same window size with a matching neighborhood in the other image. The disadvantage of this technique is its dependence on intensity values which makes it sensitive to distortions in the viewing position (perspective) as well as to changes in absolute intensity, contrast, and illumination. Also, the presence of occluding boundaries in the correlation window tends to confuse the correlation-based matcher, often giving an erroneous depth estimate. Barnard and Fischler [8] point out the relation between this type of correlation and its window size. The window size must be large enough to include enough variations of intensities for the matching and should be small enough to avoid the effect of projection distortion. Takeo in [9] resolved this issue by proposing a matching algorithm with an adaptive window size. Takeo method is used to evaluate the local variation in intensity and the disparity; followed by employing a statistical model for the disparity distribution within the window. Moravec proposed method [10] is based on the operator that computes the local maxima of a directional variance measure over a $4 \times 4$ (or $8 \times 8$ ) window around a point. The sums of squares of differences of adjacent pixels were computed along all four directions (horizontal, vertical, and two diagonals), and the minimum sum was chosen as the value returned by the operator. The site of the local maximum of the values returned by the interest operator was chosen as a feature point; this approach similar to the Sum of Squared Differences (SSD) algorithm.

The second correlation algorithms are based on feature-based matching techniques, which use symbolic features derived from intensity images rather than the image intensities. The features used are most commonly either edge points or edge segments (derived from connected edge points) that may be located with sub pixel precision. Feature based methods are more efficient than correlation-based but are more complicated in terms of coding. Prashan and Farzad [11] constructed a corresponding algorithm based on Moment invariant, through using Harris corner detection to produce reliable feature points; Maurizio in [12] used an algorithm proposed initially by Scott and Longuet [13] to find corresponding features in planar point patterns using Singular Value Decomposition SVD mathematics.

In regard to triangulation algorithms, the literature can be mainly divided into two main categories: active and passive methods. In active triangulation, a projected, structured light source is used to compute the depth value [14]. The advantage of active triangulation is in their ease of implementation. On the other hand, passive triangulation, rely on un-structured ambient light to compute the depth, making it more flexible than active methods.

Jingting et al. [15] proposed a stereo vision system that includes rectification, stereo matching, and disparity refinement, this system is realized using a single field programmable gate array, with an adaptive support weights method. Pedram et al. [16] combined stereo triangulation with matching against view set of the object to have 6 DoF post estimation of the target. This approach requires the object to be single colored and to have the pose estimation be based on their shape. The reported error is calculated in the range between 10-20 mm. Aziz and Mertsching [17] used passive stereo setup to introduce a method for quick detection of the location in stereo scenes, which contain high amount of depth saliency, using a color segmentation routine based on a seed-to-neighbor distance measurement done in hue, saturation, and intensity (HSI) color space. This method does not deliver a dense, perfect disparity map however the resulted quality is still sufficient to identify depth wise salient locations.

The proposed code implementation is in a stereo-vision setup designed for a pick and place robotic application. In autonomous pick and place there are 2D, $2.5 \mathrm{D}$ or $3 \mathrm{D}$ methods provide vision sense to the robot. In $2 \mathrm{D}$ vision systems, measurements of the $x, y$ positions are only sought while the $2.5 \mathrm{D}$ vision measures the $x, y$ coordinates and the target rotation. In the case when the 
distance between the camera and the work-piece varies arbitrary, the 2D vision system is inadequate. This motivates a more robust and accurate robot guidance system in handling applications, through 3D imaging. Many techniques exist to provide 3D vision for pick and place robotics; Wes et al. in [18] proposed the use of an active triangulation code using a 2D gray-scale camera and a laser vision sensor. Pochyly et al. [19] used a single camera along with two linear laser projectors to achieve an accuracy of about $\pm 1 \mathrm{~mm}$. However, this approach is time consuming and knowing the exact auxiliary position in this technique is very important for achieving a precise placement of an oriented object into a process station within the whole work flow. Another method proposed by Kazunori [20] used a single camera for a bin-picking application. This system can measure the 3D pose of the targets by registering the patterns that are captured from diverse view-points to the object in its initial stage. The $3 \mathrm{D}$ pose of object is estimated by comparing its position and orientation between the pre-registered patterns and the captured images. The disadvantage of such is its time consuming calculations needed to register the various patterns between the diverse positions and orientations.

Pick and place applications using stereo based methodologies have also been reported by Parril in [21], where he used passive triangulation with feature-based corresponding algorithm to find the 3D location fed into UMI robot arm to pick and place objects. The corresponding algorithm used by Parril was based on finding features from each image that are related to straight lines and circular arcs.

Tobias and George [22] proposed a pick and place application based on stereo vision technique using dual colored CCD detectors installed on both sides of the work surface, they targeted cylindrical pellets; using a color search code to help identify the object locations based on its color. On the other hand, Rahardja and Kosaka [23] developed a stereo based algorithm to find simple visual clues of complex objects. Their system required human intervention for specific grasping tasks. Martin and et al. [24] used stereo matching with structured light for an autonomous picking process while he used a set of CAD models to determine the pose of these objects. Hema et al. [25] proposed an autonomous binpicking using a stereo vision approach, where the topmost object was assumed to have highest intensity level compare to rest of objects. This methodology used the centroids to identify the $x$ and $y$ positions while the stereo images were used to train a neural network to compute the distance information.

\section{Extracting Depth Information Using Correlation Method}

This section discusses the details of the acquisition, cali- bration and depth calculation steps.

\subsection{Image Acquisition and Modeling}

In this study, the imaging device is based on two open architecture Charged Coupled Devices CCDs. The CCD sensors are set to capture monochrome images with a spatial resolution of 640 pixels across its width and 480 pixels across its length; with the two cameras are securely mounted on two stable tripods, while the targeted objects are static. The two cameras are labeled as the right and the left camera and placed to acquire the same field of view, which contained two cylinders as the targeted objects.

In camera modeling, one can employ a set of mathematical equations with the camera parameters being the main variables. The two cameras are calibrated using a 2D Plane based scheme, using a grid with check board pattern of $29 \times 29 \mathrm{~mm}$. The calibration can be done through one of two implementations, either moving the imagers while fixing the pattern or moving the pattern while fixing the imagers. In this study the second option is used.

Figure 1 displays the way that the calibration pattern is manipulated in space, from the left camera perspective. The higher the number of images that are taken for the different orientations, the higher the calibration accuracy. 32 images are captured for the calibration step; 16 images from the right camera and 16 images from the left camera.

The calibration is done using approach developed by Jean [26]. The calibration results in the camera intrinsic and extrinsic parameters. The intrinsic parameters define the internal geometric and optical characteristics of the camera mainly; its Focal length $\left(f_{c}\right)$, horizontal and vertical focal lengths $f_{c}(1), f_{c}(2)$. The $f_{c}(2) / f_{c}(1)$ is called the aspect ratio . In the proposed camera system, the aspect ratio is set to about one because the camera pixels are

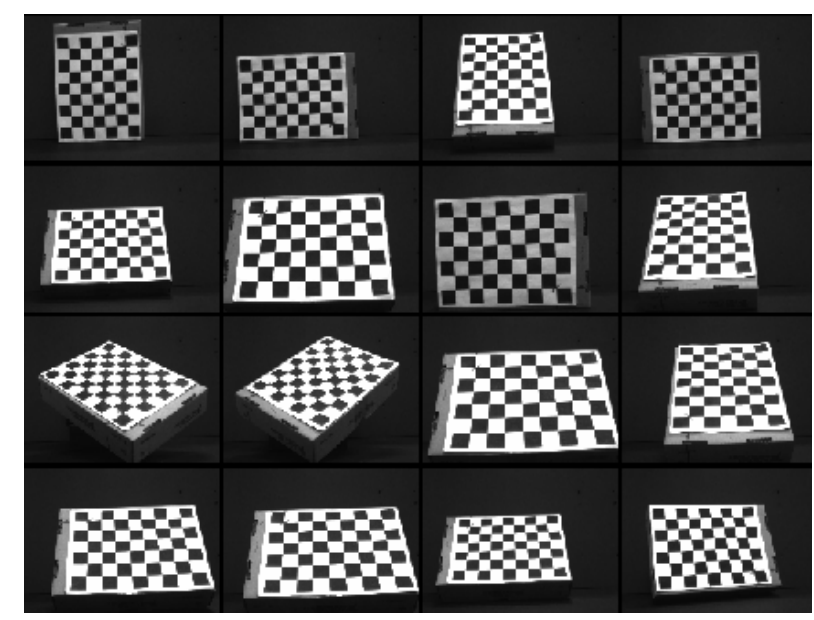

Figure 1. Left calibration images. 
square. Other intrinsic factors include the Principal point (cc) and the skew coefficient (alpha_c), which defines the angle between the $\mathrm{x}$ and $\mathrm{y}$ axes. In this case, the coefficient is zero because of the square pixels used. Additionally, the distortions $\left(k_{c}\right)$ are computed to describe the image distortion coefficients including the radial and tangential distortions. In regard to the extrinsic parameters that describe the camera position and orientation with respect to the calibration pattern, they are represented through two matrices; a rotation matrix $R$ and a translational matrix $T$. These two matrices are used to uniquely identify the transformations between the unknown camera reference frame and the known world reference frame [27]. The extrinsic parameters depend on the camera pose/orientation and unlike the intrinsic parameters, their values change once the camera pose is changed.

The calibration resulted in a pixel error around 0.21 pixels in the x-direction and around 0.45 pixels in the $\mathrm{y}$-direction. The calibration error in mm units based on pixel pitch relation is found in tabulated in Table $\mathbf{1}$.

\subsection{Correspondence Analysis}

The traditional Sum of Square Difference or SSD algorithm employs a window around each pixel to search for close resemblance in the other perspective image. The comparison of the blocks is done using Equation (1);

$$
S S D=\sum_{x y}\left[I_{T}(x, y)-I(x, y)\right]^{2}
$$

where $I(x, y)$ is pixel intensity in the first image (left image), and $I_{T}(x, y)$ is from the second image (right image). The block from the second window that has the smallest value of SSD as compared with others, is chosen as the window that closely resembles the block from the first image. The center of this block is then chosen as the corresponding point of the pixel from the first image. The SSD algorithm based on intensity comparison has been tested using images shown in Figures 2(a)-(b), with a window size of $3 \times 3$ pixels. With six points selected for the comparison as shown in Figure 3, the resulted accuracy is tabulated in Table 2 . The SSD algorithm shows good results, however following challenges are identified; firstly, the code searches all pixels for correspondence which is not ideal because not all the same pixels exist in

Table 1. Error in calibration in $\mathbf{m m}$ units.

\begin{tabular}{ccccc}
\hline & $\begin{array}{c}\text { Image Size } \\
\text { in mm units }\end{array}$ & $\begin{array}{c}\text { Image size } \\
\text { in pixels }\end{array}$ & $\begin{array}{c}\text { Error in } \\
\text { pixels }\end{array}$ & $\begin{array}{c}\text { Error in } \\
\text { mm units }\end{array}$ \\
\hline $\begin{array}{c}\text { X-direction } \\
\text { (Horizontal) }\end{array}$ & $150 \mathrm{~mm}$ & 640 pixels & 0.21 pixels & $0.05 \mathrm{~mm}$ \\
$\begin{array}{c}\text { y-direction } \\
\text { (Vertical) }\end{array}$ & $110 \mathrm{~mm}$ & 480 pixels & 0.45 pixels & $0.1 \mathrm{~mm}$ \\
\hline
\end{tabular}

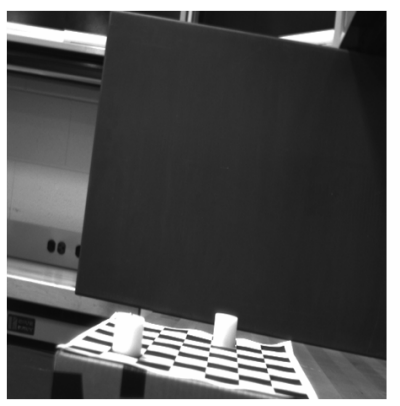

(a)

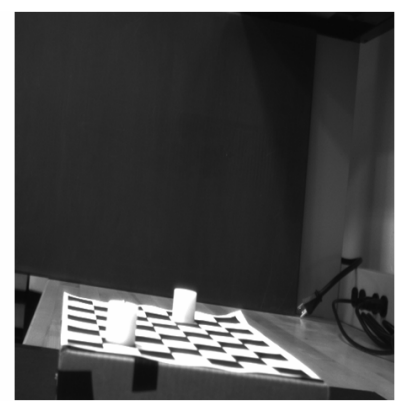

(b)
Figure 2. (a) Right image to test SSD algorithm; (b) Left image to test SSD algorithm.

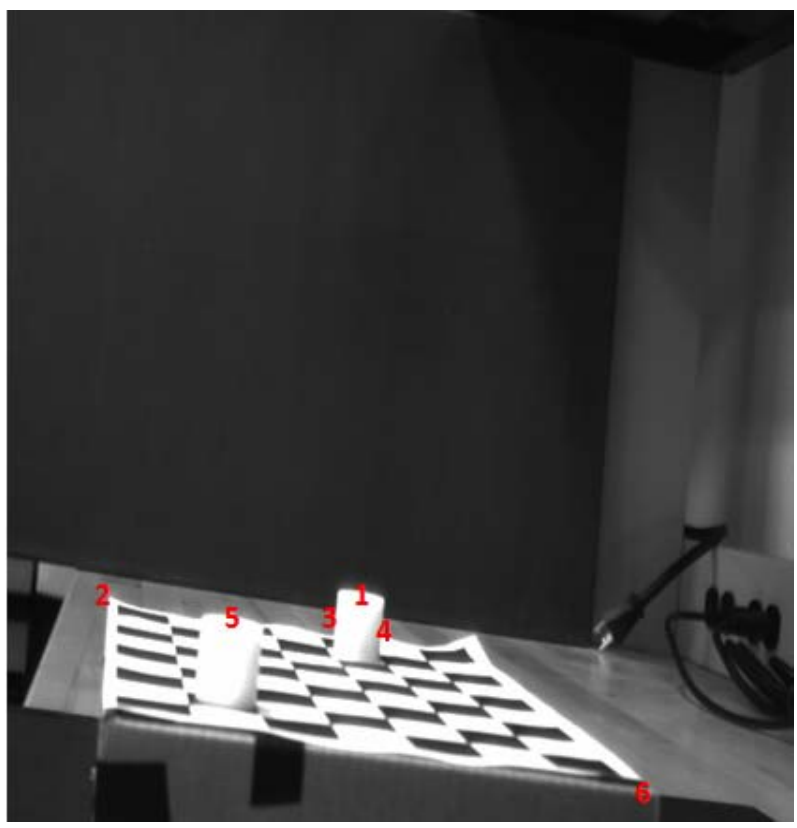

Figure 3. Testing selection points in left image.

both images that might result in a false positive [28]. The SSD searches all the pixels in the right image $640 \times 480$ pixels, leading to long processing time for each corresponding pixel, which is found to be around 2.55 seconds for a machine that runs a core i7 CPU 2.67 GHZ, RAM 4 GB.

Figures 4(a) and (b) show two outputs from the traditional SSD algorithm; one considered as the correct matching as in Figure 4(a), a while the other is a false matching case in Figure 4(b). Where the $\mathrm{x}$-axis is the $y$ coordinate of right image and $\mathrm{y}$-axis is the minimum SSD. To minimize the high number of incorrect matching, a modified SSD algorithm is developed and further discussed in Section 3.3.

\subsection{Modified SSD Algorithm}

This study applies three modifications to the original SSD algorithm to improve its accuracy and reduce the 
Table 2. SSD test results.

\begin{tabular}{|c|c|c|c|c|c|c|c|}
\hline \multirow{2}{*}{ Points tag } & \multicolumn{2}{|c|}{$\begin{array}{l}\text { Points coordinate } \\
\text { in left image }\end{array}$} & \multicolumn{2}{|c|}{$\begin{array}{l}\text { Manually estimated position } \\
\text { in right image }\end{array}$} & \multicolumn{2}{|c|}{$\begin{array}{l}\text { Calculated coordinate } \\
\text { using SSD algorithm }\end{array}$} & \multirow{2}{*}{ Comments } \\
\hline & $\mathrm{X}$ & $\mathrm{Y}$ & $\mathrm{X}$ & $\mathrm{Y}$ & $\mathrm{X}$ & $\mathrm{Y}$ & \\
\hline 1 & 357 & 300 & 357 & 408 & 357 & 411 & Good correlation \\
\hline 2 & 387 & 108 & 387 & 220 & 387 & 223 & Good correlation \\
\hline 3 & 375 & 285 & 375 & 393 & 375 & 391 & Good correlation \\
\hline 4 & 375 & 315 & 375 & 423 & 375 & 417 & Good correlation \\
\hline 5 & 382 & 203 & 382 & 246 & 382 & 368 & False correlation \\
\hline 6 & 430 & 542 & \multicolumn{2}{|c|}{ Does not exist in right image } & 430 & 265 & False correlation \\
\hline
\end{tabular}

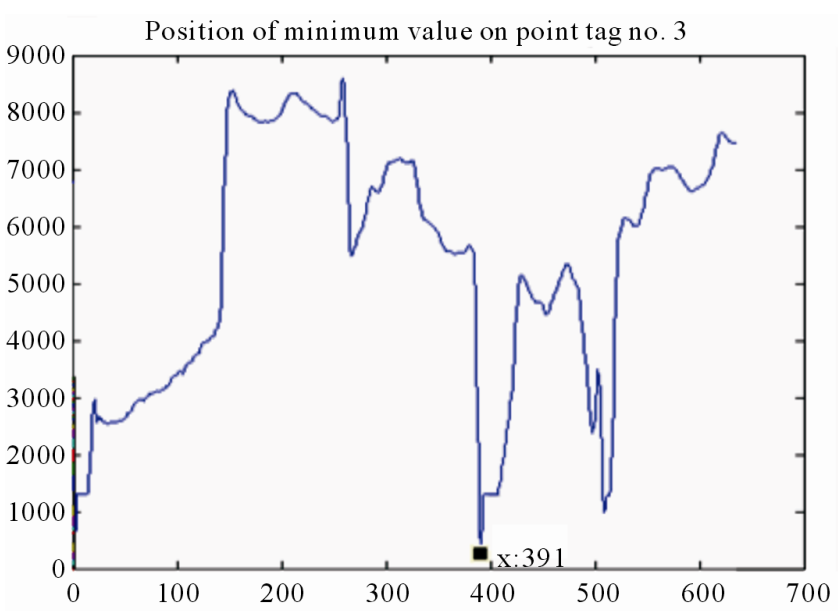

(a)

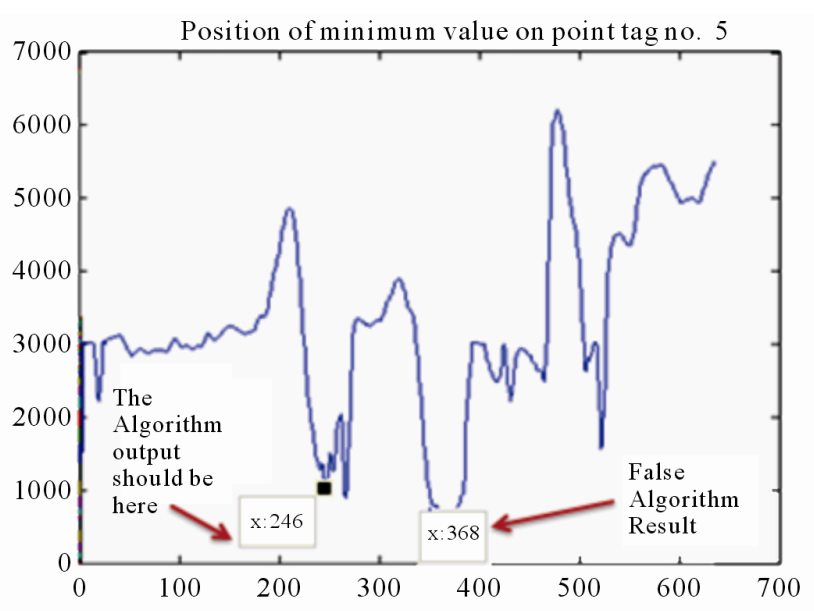

(b)

Figure 4. (a) Good correlation related to point tag No. 3; (b) False correlation related to point tag No. 5.

processing time. First change is done by implementing a specific Field of View for the search, by recognizing and establishing the region of interest within each image where the value-added information (objects) exists. In Figure 5, the new ROI is highlighted with a red border.

Second change is done by guiding the SSD algorithm to search for detected objects' Centroids. This modification can be implemented by threshold the image thusly removing the background (non-value added) information, in addition to filtering the resulted binary image to further remove data noise. Also, a combination of morphological operations namely; erosion and dilation are done to help define the detected objects' boundaries. Finally, the centroid can be readily detected as in Figure 6 .

The third modification to the traditional SSD is done through a Rectification step. The rectification process determines a transformation of each image plane such that the pairs of conjugate epipolar lines become collinear and parallel to one of the image axes.

The distinct advantage of rectification is that computing stereo correspondences more efficient, because the

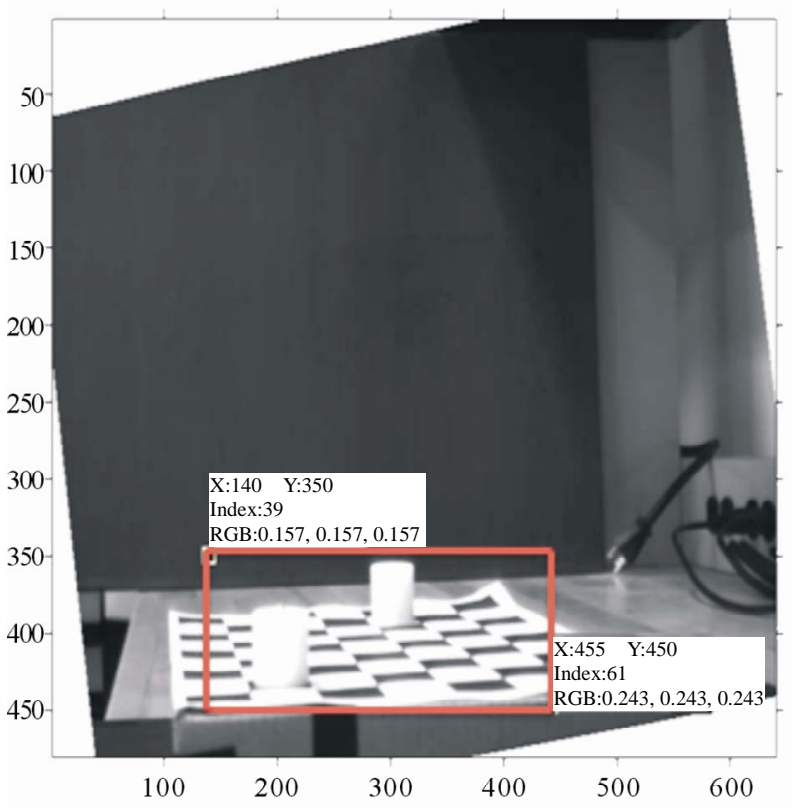

Figure 5. Dynamic ROI implementation. 

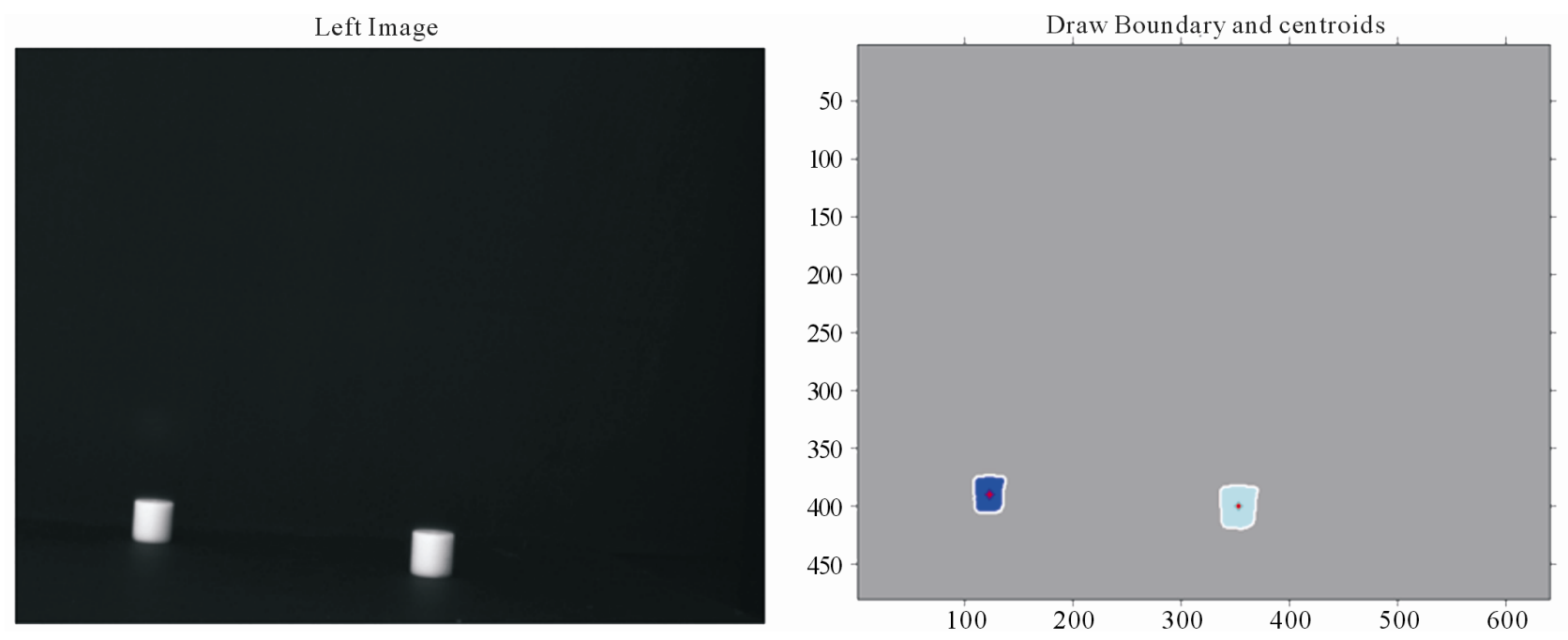

Figure 6. (a) Left image; (b) Objects' boundaries and centroids.

search need only to consider the horizontal lines of the rectified images, reducing the search domain from $2 \mathrm{D}$ to 1D. The calibration step should be done before the rectification because the internal parameters should be known in this stage. The rectification [29] defines new Perspective Projection Matrix PPM by rotating the old ones around their optical centers until the focal planes become coplanar, thereby containing the baseline. This ensures that the epipoles are at infinity; hence, epipolar lines are parallel. To have horizontal epipolar lines, the baseline must be parallel to the new x-axis of both cameras. In addition, to have a proper rectification, conjugate points must have the same vertical coordinate. The Perspective Projection Matrix PPM is found through the equation (2) below.

$$
P P M=K K \cdot[R \backslash T]
$$

where, $R$ is the rotational matrix, $T$ is the translational matrix which found as extrinsic parameters in calibration step.

The camera matrix $K K$ contains intrinsic parameters which found in calibration step.

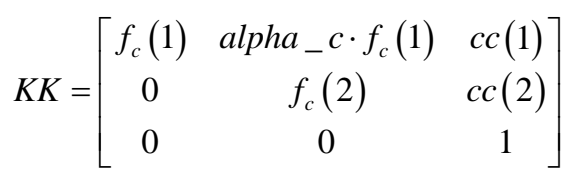

The result of rectification step is found in Figures 7(a) and (b).

The results from the modified SSD code are in Table $\mathbf{3}$ showing good results in term of accuracy and resource efficiency. The error values are around 1.49 pixels in the $\mathrm{x}$-direction and 2.72 pixels in the $\mathrm{y}$-direction.

\subsection{Triangulation}

This section discusses the Integration of the modified
SSD algorithm along with the triangulation step. The triangulation is done in a passive mode to allow for more flexibility and robustness in terms of ambient lighting. Furthermore, the triangulation follows a linear square solution approach reported in [26], which is usefully summarized in following equations; suppose the coordinates for first cylinder in the right camera reference frame as;

$$
P_{R}=\left[\begin{array}{c}
X_{R} \\
Y_{R} \\
Z_{R}
\end{array}\right]
$$

And the coordinates for first cylinder in the left camera reference frame as;

$$
P_{L}=\left[\begin{array}{c}
X_{L} \\
Y_{L} \\
Z_{L}
\end{array}\right]
$$

After the calibration, the extrinsic parameters like rotation matrix and translational matrix are known. Then the relation between $P_{R}$ and $P_{L}$ is;

$$
P_{L}=R \cdot P_{R}+T
$$

Also, the projective of the two points $p_{l}$ and $p_{r}$ on the image plane is their perspective projection. Retrieving the $P_{L}$ and $P_{R}$ from the coordinates of the $p_{l}$ and $p_{r}$ results in;

$$
\begin{gathered}
p_{r}=\frac{P_{R}}{Z_{R}}=\left[\begin{array}{c}
x_{R} \\
y_{R} \\
1
\end{array}\right] \\
p_{l}=\frac{P_{L}}{Z_{L}}=\left[\begin{array}{c}
x_{L} \\
y_{L} \\
1
\end{array}\right]
\end{gathered}
$$




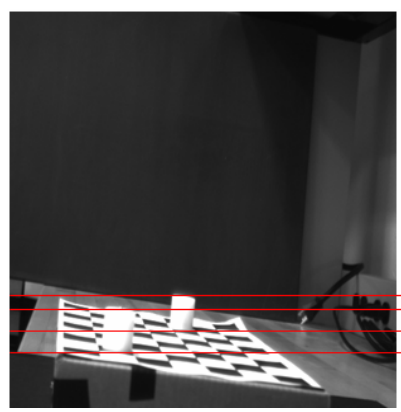

Left Image (original)

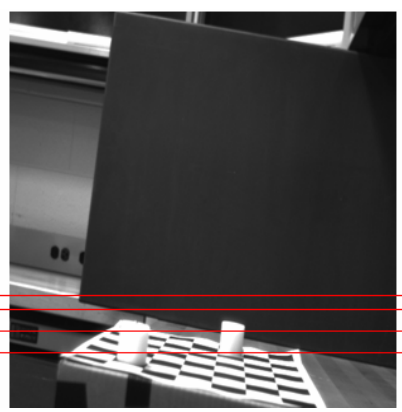

Right Image (original)

(a)

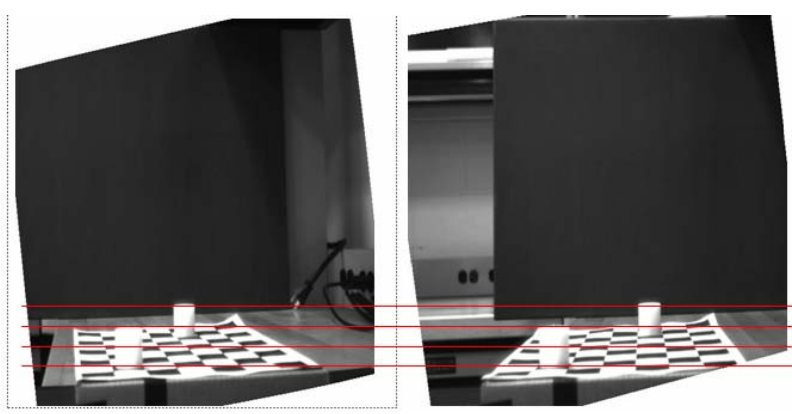

Rectified Left Image

(b)

Figure 7. (a) Right and left image before rectification step; (b) Right and left image after rectification step.

Table 3. Results of modified SSD.

\begin{tabular}{cccc}
\hline & Actual Centroids for Two Cylinders & Centroids using Modified SSD Algorithm & The Maximum Error in Pixels \\
\hline \multirow{2}{*}{ Left Image } & $(389.9,123.7)$ & $(390,124)$ & 0.28 \\
& $(400.7,354.0)$ & $(401,354)$ & 1.49 in x-direction and 2.72 in y-direction \\
Right Image & $(388.1,231.6)$ & $(390,231)$ & \\
Elapsed time & $(399.5,442.7)$ & $(401,440)$ & \\
\hline
\end{tabular}

By instituting these relations to the following Equation (9); results in Equation (10); upon re-arrangement becomes Equation (11)

$$
\begin{aligned}
& P_{L}=R P_{R}+T \\
& Z_{L} \cdot P_{l}=Z_{R} \cdot R \cdot p_{r}+T
\end{aligned}
$$

Rearrange the previous equation to:

$$
\left[\begin{array}{ll}
-R \cdot p_{r} & p_{l}
\end{array}\right]\left[\begin{array}{l}
Z_{R} \\
Z_{L}
\end{array}\right]=T
$$

where, $A=\left[-R \cdot p_{r} p_{l}\right]$ is a matrix of $3 \times 2$; using the least square solution to (11), yields Equation (12);

$$
\left[\begin{array}{c}
Z_{R} \\
Z_{L}
\end{array}\right]=\left(A^{T} A\right)^{-1} A^{T} T
$$

where, $\alpha=-R p_{r}$ With the final equation re-arranged and written as (13) with $<>$ representing a standard scalar product operator.

$$
Z_{R}=\frac{\left\|p_{l}\right\|^{2}\langle\alpha, T\rangle-\left\langle\alpha, p_{l}\right\rangle\left\langle p_{l}, T\right\rangle}{\|\alpha\|^{2}\left\|p_{l}\right\|^{2}-\left\langle\alpha, p_{l}\right\rangle^{2}}
$$

where, $p_{l}=\left[\begin{array}{cccc}x_{1} & x_{2} & \cdots & \cdots \\ y_{1} & y_{2} & \cdots & \cdots\end{array}\right]$, this is the $x$ and $y$ coordinates of all objects Centroids in the left image and $p_{r}=\left[\begin{array}{cccc}x_{1} & x_{2} & \cdots & \cdots \\ y_{1} & y_{2} & \cdots & \cdots\end{array}\right]$, this is the corresponding points found through modified SSD algorithm.

\section{Results}

The flow chart illustrated in Figure 8 displays the steps followed when the proposed SSD algorithm is implemented.

To test the stereo vision implementation with modified SSD algorithm, two experiments are done. The first experiment setup is shown in Figure 9, where two cylinders are placed in front of the left and the right cameras while their depths are calculated.

The optimal baseline for this experiment is around 20 $\mathrm{cm}$, the close cylinder is placed around $90 \mathrm{~cm}$ from the cameras' plane with the experiment repeated 7 times with new cylinder location for each time. The results are tabulated in Table 4, showing good agreement between the actual and the reported depths; the average depth error is found to be around $1.2 \mathrm{~mm}$ while the maximum error in depth is around $2.9 \mathrm{~mm}$.

The second experiment is an implementation of an autonomous pick and place exercise. For this exercise, the goal is to compute the distance between the robotic gripper and the targeted objects. This is done by detecting the pick-up location for each object while comparing it to a ground reference so that the system can calculate the height of each acquisition. Additionally the targeted objects are arranged in a stack configuration that resembles real-life scenarios. The proposed setup calculates the depths to enable the gripper to accurately adjust for each object height and depth. Two CCD detectors are placed in a stereo-vision configuration while the gripper is placed (reference location) to be in the midpoint between the two detectors, on same base line. This setup is illustrated in Figure 10.

The complete procedure is repeated several times to assess the system repeatability with changing the camera 


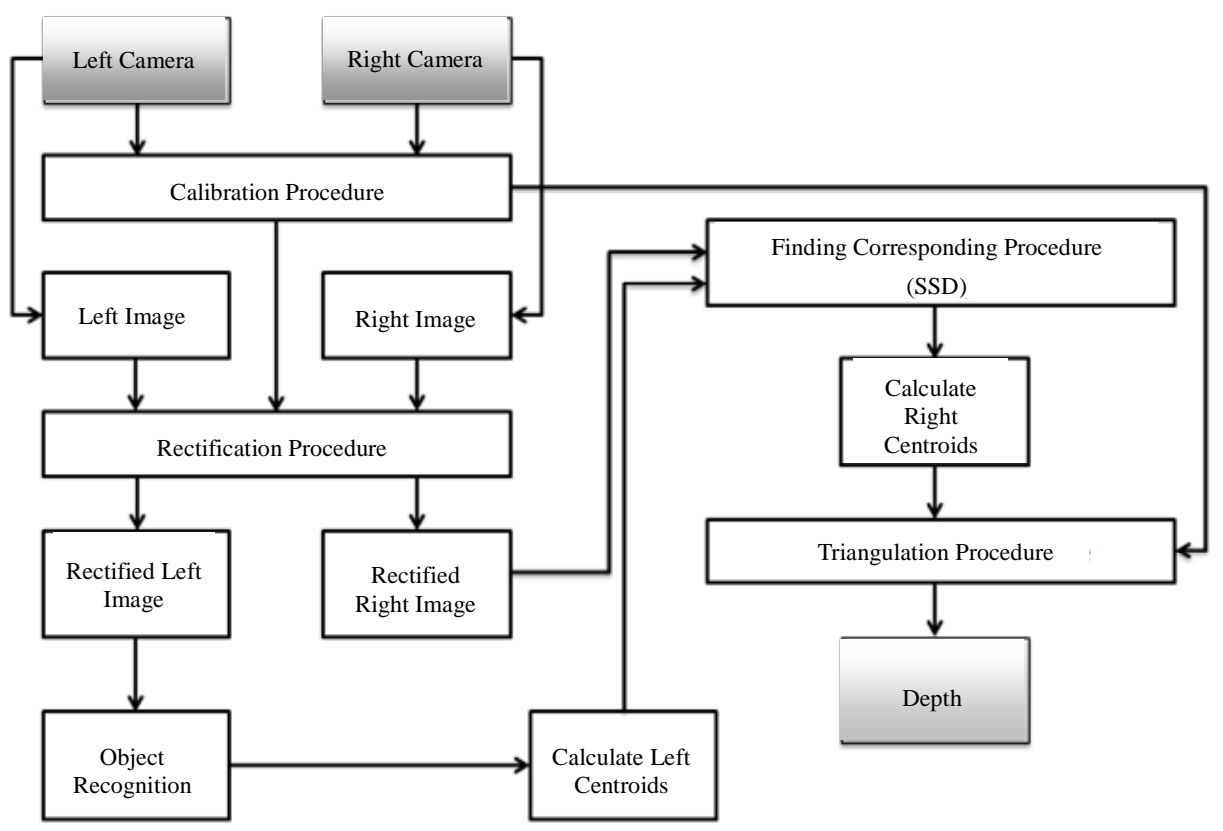

Figure 8. Flow chart to find triangulation using modified SSD algorithm.

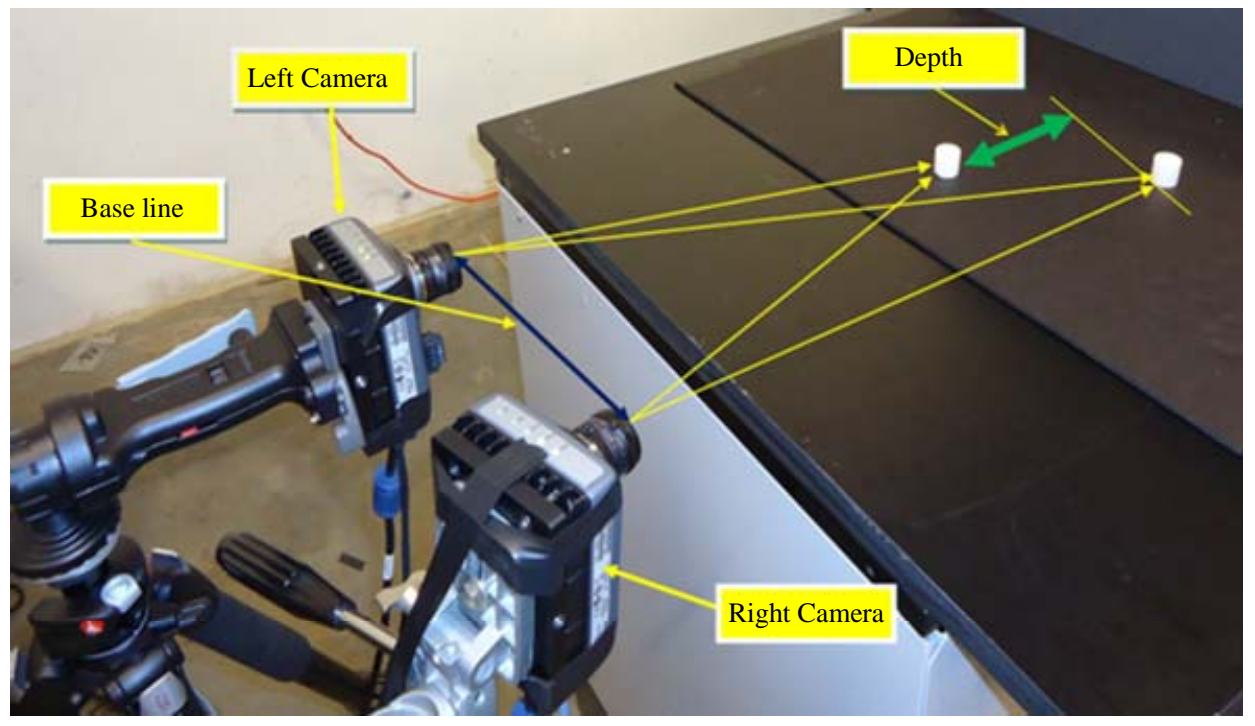

Figure 9. Triangulation based on stereo vision.

Table 4. Triangulation results with baseline equal $20 \mathrm{~cm}$.

\begin{tabular}{ccccc}
\hline Case No. & Actual Depth in cm unit & Calculated Depth in cm unit & Depth Error in cm & Percentage Error (\%) \\
\hline Case 1 & 10.00 & 10.10 & 0.1 & 1.00 \\
Case 2 & 6.50 & 6.43 & 0.07 & 1.08 \\
Case 3 & 19.00 & 18.83 & 0.17 & 0.89 \\
Case 4 & 15.50 & 15.43 & 0.07 & 0.45 \\
Case 5 & 12.50 & 12.37 & 0.13 & 1.04 \\
Case 6 & 3.50 & 2.21 & 0.29 & 8.29 \\
Case 7 & 24.00 & & 0.04 & 0.17 \\
& Maximum Depth Error in cm & & 0.29 & 0.04 \\
& Minimum Depth Error in cm & & 0.12 & \\
\hline
\end{tabular}


location; the rectification results are further displayed in Figures 11 and 12.

The detected objects' boundaries and their Centroids detection are modified to pinpoint two points; the pickup location and the reference ground point. Having these two locations identified, enable the code to compute the distances which are then sent to the gripper for actuation. This exercise is repeated six times using different number of objects stacked on top of each other. Figure $\mathbf{1 3}$ shows the objects' boundaries and the pick up location detection identified.

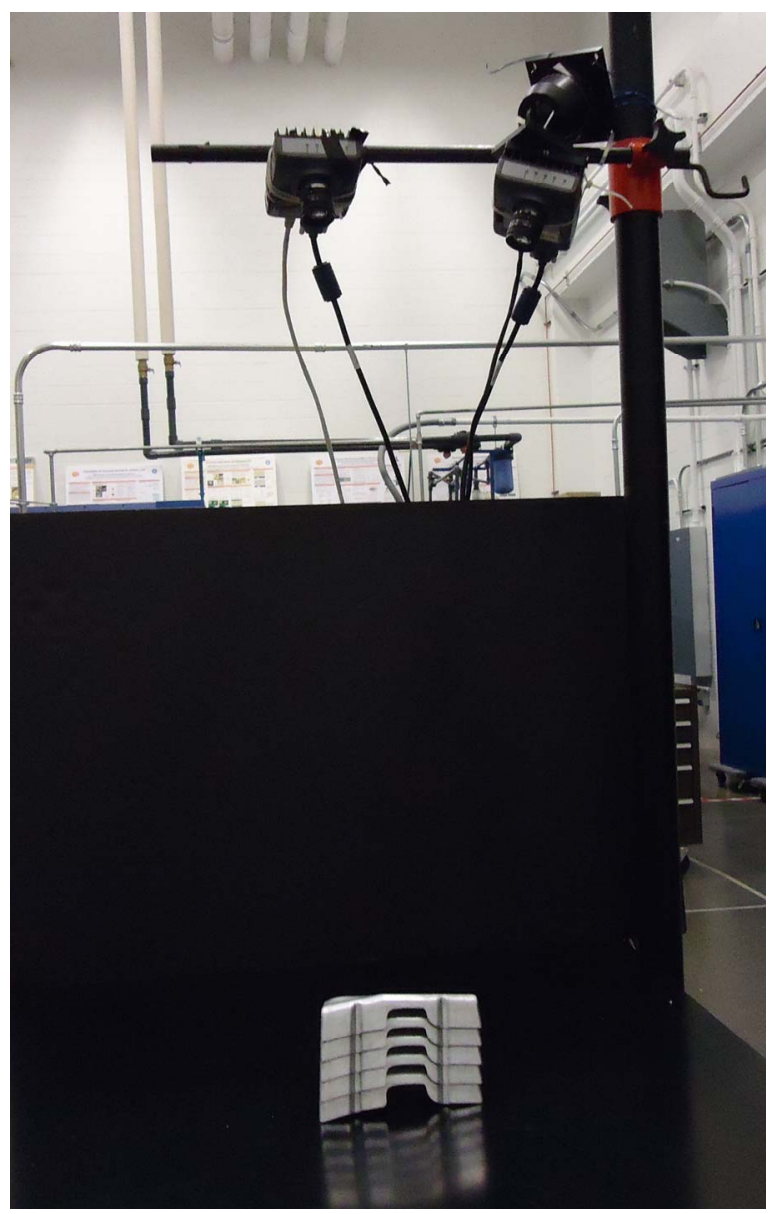

Figure 10. Triangulation based on stereo vision (Second experiment).

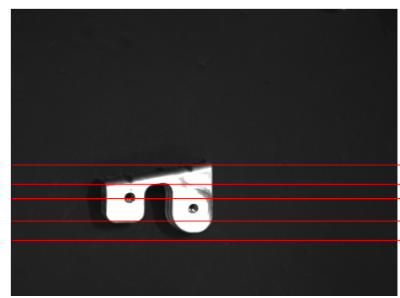

(a)

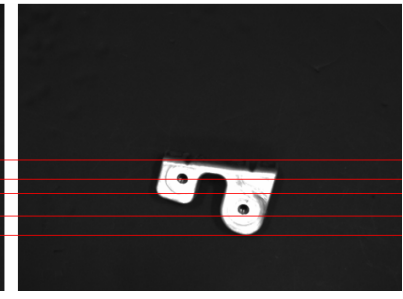

(b)
Figure 11. Before rectification: (a) Left image; (b) Right image.

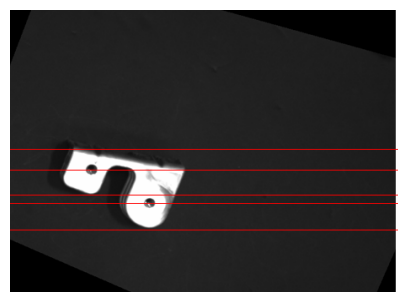

(a)

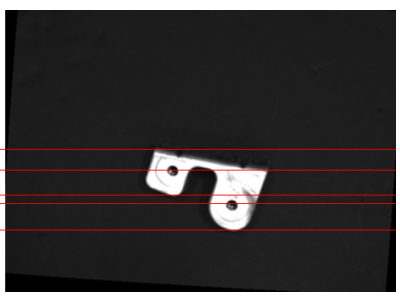

(b)
Figure 12. After rectification: (a) Left image; (b) Right image.

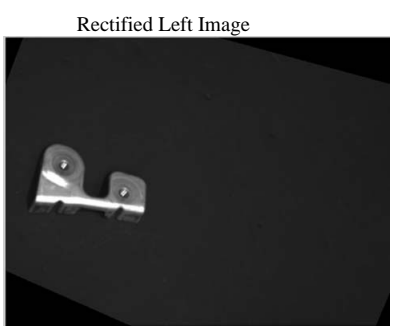

(a)

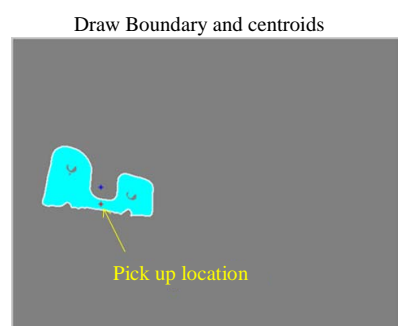

(b)
Figure 13. (a) Rectified left image; (b) Objects' boundaries and pickup location.

\section{Conclusion}

The presented study proposed a modified, efficient SSD algorithm that can be used within the context of stereo-triangulation for autonomous pick and place. The suggested modifications were in two folds; reducing the search domain by establishing a ROI for the value-added data and through a rectification step that is done to reduce the 2D search domain into 1D. The second fold relied on the use of detected objects' centroids rather than their pixels' intensities. Thusly is improving the code robustness against any changes in the surroundings' illumination. The developed code results in better accuracy and repeatability, without any false positives at faster processing times. An autonomous pick and place exercise is also demonstrated using the developed algorithm, future work includes further refinement to the SSD code to improve its processing efficiency and robustness.

\section{REFERENCES}

[1] C. Tyler, "Binocular Vision,” Foundations of Clinical Ophthalmology, Vol. 2, Chapter 24, 1982.

http://www.ski.org/CWTyler_lab/CWTyler/TylerPDFs/T yler_BinocVisionDuanes2004.pdf

[2] G. DeAngelis, B. Cumming and W. Newsome, "Cortical Area MT and the Perception of Stereoscopic Depth,” Journal Nature, Vol. 394, 1998, pp. 677-680.

[3] R. Kletter, K. Schluns and A. Koschan, "Three-Dimensional Data from Images,” 2nd Edition, Springer-Verlag Singapore Pte. Ltd., Singapore, 1998.

[4] R. Y. Tsai, “A Versatile Camera Calibration Technique for 
High-Accuracy 3D Machine Vision Metrology Using of the Shelf TV Cameras and Lenses," IEEE Journal of Robotics and Automation, Vol. 3, No. 4, 1987, pp. 323-344. doi:10.1109/JRA.1987.1087109

[5] O. Faugeras, “Three-Dimensional Computer Vision: A Geometric Viewpoint,” MIT Press, Cambridge, 1993.

[6] Z. Zhang, "A Flexible New Technique for Camera Calibration,” IEEE Transactions Pattern Analysis and Machine Intelligence, Vol. 22, No. 11, 2000, pp. 1330-1334. doi:10.1109/34.888718

[7] Z. Zhang, "Camera Calibration with One Dimensional Objects,” IEEE Transactions Pattern Analysis and Machine Intelligence, Vol. 26, No. 7, 2004, pp. 892-899.

[8] S. T. Barnard and M. A. Fischler, "Stereo Vision," Encyclopedia of Artificial Intelligence, John Wiley, New York, 1987, pp. 1083-1090.

[9] T. Kanade and M. Okutomi, "A Stereo Matching Algorithm with Adaptive Window: Theory and Experiment," IEEE Transactions on Pattern Analysis and Machine Intelligence, Vol. 16, No. 9, 1994, pp. 869-881. doi:10.1109/34.310690

[10] H. Moravec, "Towards Automatic Visual Obstacle Avoidance," Proceedings of the 5th International Joint Conference on Artificial Intelligence, Cambridge, 22-25 August 1977, p. 584.

[11] P. Premaratne and F. Safaei, "Stereo Correspondence Using Moment Invariants," Communications in Computer and Information Science, Vol. 15, No. 12, 2008, pp. 447-454. doi:10.1007/978-3-540-85930-7_57

[12] M. Pilu, "A Direct Method for Stereo Correspondence Based on Singular Value Decomposition," Proceedings of 1997 IEEE Computer Society Conference on Computer Vision and Pattern Recognition, San Juan, 17-19 June 1997, pp. 261-266.

[13] G. L. Scott and H. C. Longuet-Higgins, "An Algorithm for Associating the Features for Two Patterns," Proceedings of Royal Society London, Vol. B244, No. 1309, 1991, pp. 21-26. doi:10.1098/rspb.1991.0045

[14] M. Hebert, "Active and Passive Range Sensing for Robotics," Proceedings of the 2000 IEEE International Conference on Robotics \& Automation, San Francisco, 24-28 April 2000, pp. 102-110.

[15] D. Jingting, L. Jilin, Z. Wenhui, Y. Haibin, W. Yanchang and G. Xiaojin, "Real-time Stereo Vision System Using Adaptive Weight Cost Aggregation Approach,” EURASIP Journal on Image and Video Processing, Vol. 2011, No. 20, 2011, pp. 119. doi:10.1186/1687-5281-2011-20

[16] P. Azad, T. Asfour and R. Dillmann, “Accurate Shape-Based 6-DoF Pose Estimation of Single-Colored Objects," The 2009 IEEE/RSJ International Conference on Intelligent Ro- bots and Systems, St. Louis, 11-15 October 2009, pp. 2690-2695.

[17] M. Zaheer and B. Mertsching, "Pre-Attentive Detection of Depth Saliency Using Stereo Vision,” 2010 IEEE 39th Applied Imagery Pattern Recognition Workshop AIPR, 2010, pp. 1-7.

[18] W. Iversen, "Vision-Guided Robotics: In Search of the Holy Grail,” Automation World, 2006, pp. 28-31. http://www.automationworld.com/feature-1878

[19] A. Pochyly, T. Kubela, M. and P. Cihak, "Robotic Vision for Bin-Picking Applications of Various Objects,” 2010 41st International Symposium on and 2010 6th German Conference on Robotics, Munich, 7-9 June 2010, pp. 1-5.

[20] B. Kazunori, W. Fumikazu, K. Ichiro and K. Hidetoshi, "Industrial Intelligent Robot,” FANUC Technology Review Journal, Vol. 16, No. 2, 2003, pp. 35-41.

[21] J. Porrill, S. B. Pollard, T. P. Pridmore, J. B. Bowen, et al., “TINA: A 3D Vision System for Pick and Place," Image and Vision Computing, Vol. 6, No. 2, 1988, pp. 65-72.

[22] T. Kotthäuser and F. Georg, "Vision-Based Autonomous Robot Control for Pick and Place Operations," 2009 IEEE/ ASME International Conference on Advanced Intelligent Mechatronics Suntec Convention and Exhibition Center, Singapore, 1417 July 2009, pp. 1851-1855.

[23] K. Rahardja and A. Kosaka, "Vision-Based Bin-Picking: Recognition and Localization of Multiple Complex Objects Using Simple Visual Cues,” Proceedings of IEEE/RSJ International Conference on Intelligent Robots and Systems, 4-8 October 1996, pp. 1448-1457.

[24] B. Martin, B. Bachler and S. Stefan, "Vision Guided Bin Picking and Mounting in a Flexible Assembly Cell," Thirteenth International Conference on Industrial and Engineering Application of Artificial Intelligence and Expert Systems, New Orleans, 19-20 June 2000, pp. 255-321.

[25] H. Chengalvarayan, P. Murugesapandian, N. Ramachandran and S. Yaacob, "Stereo Vision System for A Bin Picking Adept Robot," Malaysian Journal of Computer Science, Vol. 20, No. 1, 2007, pp. 91-98.

[26] J. Bouguet, "Camera Calibration Toolbox for Matlab," 2010. http://www.vision.caltech.edu/bouguetj/calib_doc/

[27] E. Trucco and A. Verri, "Introductory Techniques for 3-D Computer Vision,” Prentice-Hall, 1998.

[28] N. Ayache and P. Sander, "Artificial Vision for Mobile Robots: Stereo Vision and Multisensory Perception,” MIT Press, Cambridge, 1991.

[29] A. Fusiello, E. Trucco and A. Verri, "A Compact Algorithm for Rectification of Stereo Pairs," Machine Vision and Applications, Vol. 12, No.1, 2000, pp. 16-22. doi:10.1007/s001380050120 